\title{
Utility of a Bayesian Mathematical Model to Predict the Impact of Immunogenicity on Pharmacokinetics of Therapeutic Proteins
}

\author{
Steven Kathman Jr., ${ }^{1}$ Theingi M. Thway, ${ }^{2}$ Lei Zhou, ${ }^{1}$ Stephanie Lee, ${ }^{3}$ Steven Yu$^{2}{ }^{2}$ Mark Ma, \\ Naren Chirmule, ${ }^{3}$ and Vibha Jawa ${ }^{3,4}$
}

Received 1 June 2015; accepted 30 November 2015; published online 19 January 2016

\begin{abstract}
The impact of an anti-drug antibody (ADA) response on pharmacokinetic (PK) of a therapeutic protein (TP) requires an in-depth understanding of both PK parameters and ADA characteristics. The ADA and PK bioanalytical assays have technical limitations due to high circulating levels of TP and ADA, respectively, hence, significantly hindering the interpretation of this assessment. The goal of this study was to develop a population-based modeling and simulation approach that can identify a more relevant PK parameter associated with ADA-mediated clearance. The concentrationtime data from a single dose PK study using five monoclonal antibodies were modeled using a noncompartmental analysis (NCA), one-compartmental, and two-compartmental Michaelis-Menten kinetic model (MMK). A novel PK parameter termed change in clearance time of the TP $(\alpha)$ derived from the MMK model could predict variations in $\alpha$ much earlier than the time points when ADA could be bioanalytically detectable. The model could also identify subjects that might have been potentially identified as false negative due to interference of TP with ADA detection. While NCA and onecompartment models can estimate loss of exposures, and changes in clearance, the two-compartment model provides this additional ability to predict that loss of exposure by means of $\alpha$. Modeling data from this study showed that the two-compartment model along with the conventional modeling approaches can help predict the impact of ADA response in the absence of relevant ADA data.
\end{abstract}

KEY WORDS: anti-drug antibodies (ADA); biologics; immunogenicity; impact assessment; modeling; PK/PD; population-based modeling; simulation; statistics; therapeutic proteins.

\section{INTRODUCTION}

Prediction of clinically relevant events is an essential component of drug development that can reduce program risks, significantly shrink cost, and influence the making of affordable medicines. We have developed a modeling and simulation approach to demonstrate the impact of anti-drug antibody (ADA) on pharmacokinetics (PK) of therapeutic proteins. A thorough understanding of dose-exposure and efficacy relationships is an integral part of any therapeutic protein development. The changes in PK profile of a therapeutic protein can be caused either by target-mediated drug disposition (TMDD) $(1,2)$ or due to an increased clearance caused by ADA responses to the TP (3). Not all ADA induced by the TP has an impact on drug clearance. We and others have previously reported the characteristics of ADA that have the highest likelihood of impacting clearance

\footnotetext{
${ }^{1}$ Global Biostatistical Science, Amgen Inc., One Amgen Center Drive, Thousand Oaks, California 91320, USA.

${ }^{2}$ Pharmacokinetic and Drug Metabolism Department, Amgen Inc., One Amgen Center Drive, Thousand Oaks, California 91320, USA.

${ }^{3}$ Clinical Immunology, Medical Sciences, Amgen Inc., One Amgen Center Drive, Thousand Oaks, California 91320, USA.

${ }^{4}$ To whom correspondence should be addressed. (e-mail: vibha.jawa@amgen.com)
}

and safety. These attributes include the nature (titer), kinetics, and type (isotype, neutralizing etc.) of ADA. Several factors can contribute to the inaccuracy of assessment of impact of ADA on PK of a therapeutic protein including (i) interference in the analytical measurement of TP due to excess ADA levels in a sample (4), (ii) presence of ADA in a sample which can either bind at the idiotypic region (complementarity determining region, CDR) or the framework $(\mathrm{Fc})$ region $(5)$ and interfere with accurate measurement of TP. The in-depth delineation of this interplay between ADA and PK interactions that can have a significant clinical impact is still evolving. Recent work from other groups has also demonstrated ADA-mediated clearance as a covariate in PK models $(3,6-12)$.

The primary objective of this work was to examine the utility of population-based modeling and simulation approach to predict the impact of ADA on PK. This process involved development of statistically predefined criteria that can be used for inclusion and exclusion of subjects that develop clinically relevant ADA for exposure and dose finding analyses. The methodology described also improves the accuracy of the assessment of impact of ADA on PK.

In this study, PK data from five variants of a monoclonal antibody therapeutic (to the same target) was applied to develop a statistical model to delineate the effects of ADA on PK parameters, (AUC, Cmax). The statistician was blinded to 
the ADA data set at the time of the model development. After the model was developed, the impact of a characterized ADA response on the PK of Mabs was evaluated retrospectively. The ADA characterization included evaluation of magnitude of ADA, onset of response, and their epitope specificity. Population-based PK modeling demonstrated that the change in time of clearance of these mAbs was associated with an onset of ADA response. This study proposes for the first time that the PK data can be modeled to predict clinically relevant ADA that impacts TP exposure through the novel parameter described in this work.

\section{METHODS}

\section{Study Design}

Samples from a single dose PK study in cynomolgus monkeys were obtained for ADA and PK analyses. Thirty cynomolgus monkeys were administered a single dose of $4 \mathrm{mg} / \mathrm{kg}$ intravenously with five fully human monoclonal antibody clones designated as mAbs 1 to 5 . Each clone variant had a different CDR sequence and binding affinity to the target, but identical framework sequences. The serum samples for PK assessments were collected at pre-dose and 0.5 h, 48 h (day 2), 96 h (day 4), 168 h (day 7), 240 h (day 10), 336 h (day 14), 504 h (day 21), 672 h (day 28), 840 h (day 35), $1008 \mathrm{~h}$ (day 42), and $1176 \mathrm{~h}$ (day 49) post-dose. The serum samples for ADA assessments were collected at pre-dose/ day-7 and day 7 (168 h), day 14 (336 h), day 21 (504 h), and day 28 (672 h) post-dose. All serum samples were stored at -60 to $-80^{\circ} \mathrm{C}$ until analysis. The time in hours is represented interchangeably as "h" or "hr" in the text and figures.

\section{ADA Immunoassay: Universal Indirect Species-Specific Assay}

The ADA assessments were performed using the universal indirect species-specific assay (UNISA) as described previously (4). Briefly, a bare standard-bind MSD 6000 plate was coated overnight with therapeutic antibody. Serum samples diluted 1:200 in assay buffer were either left untreated for the screening test or treated with excess relevant or irrelevant therapeutic for specificity analysis or competitive binding test. The antibody coated plates were washed after a blocking step, and diluted serum sample were added and incubated for approximately $3 \mathrm{~h}$. Plates were washed and ruthenylated mouse anti-cynomolgus monkey IgG (whole molecule) (Texas Labs) was added. After approximately $30 \mathrm{~min}$, plates were washed and $2 \times$ MSD T Read buffer was added. Lastly, plates were read using the MSD 6000 plate reader, and the response measured as electrochemiluminescence (ECL) units. Pooled normal cynomolgus monkey serum (PNCS) at 0,100 , or $500 \mathrm{ng} / \mathrm{mL}$ of cyno anti-human IgG chimeric antibody were analyzed on every plate as quality controls. For the screening assay, sample results were expressed as a ratio of the sample ECL over the negative control ECL (PNCS) as a signal to noise (S/ $\mathrm{N})$. For the specificity analysis, the ratio of the treated $\mathrm{S} / \mathrm{N}$ compared to the untreated $\mathrm{S} / \mathrm{N}$ was expressed as a percent depletion. No acid dissociation was performed for this assay format.

\section{Enzyme-Linked Immunosorbent Assay Method for Measuring Unbound MAbs Concentration}

The unbound MAb levels were quantified using a colorimetric enzyme-linked immunosorbent assay (ELISA). The 96-well microplates (Costar) were passively coated with the recombinant target protein receptor of mAbs (Amgen, Inc., CA). The mAbs in cynomolgus serum were detected with a HRP-labeled mouse monoclonal antibody against antihuman IgG Fc antibody (Amgen, Inc. CA). The range of this analytical method was $0.391-400 \mathrm{ng} / \mathrm{mL}$.

\section{PK Parameter Estimations and Modeling}

Non-compartmental and one compartmental analysis were performed on the serum concentration-nominal time data from individual animals using Phoenix ${ }^{\circledR}$ (Enterprise version 5.1.1, 2006, Pharsight ${ }^{\circledR}$ Corp, Mountain View, CA). PK parameter estimates like $\left(\mathrm{T}_{1 / 2}, \mathrm{~T}_{\text {last }}, \mathrm{C}_{\text {last }}\right.$ (concentration at last time point), clearance (CL), area under the curve (AUC)) were estimated for each animal. The mean PK estimates were calculated between ADA positive and negative animals.

A two-compartment simplified target-mediated disposition model derived from a version of population PK model described earlier (13) was developed for evaluating impact of ADA on PK. The non-linear clearance effects were evaluated using Michaelis-Menten kinetic model. This model allowed for the linear clearance to change at some point in time, presumably due to the ADA formation, and was set up to look if there was an "effect" with some probability. This analysis was accomplished by utilizing a mixture distribution for $\alpha$ (the time at which the clearance changes). If the probability of an effect (or change in clearance) is 0 , then this model would just reduce to the simplified target-mediated disposition model. The differential equation for the central compartment of this model is as follows:

$$
\begin{array}{r}
\mathrm{dC} / \mathrm{dt}=\operatorname{Input}-(\mathrm{CL} * \mathrm{I}(\mathrm{t}<\alpha)+\eta * \mathrm{I}(\mathrm{t}>\alpha)) * \mathrm{C} / \mathrm{V} \\
-(\operatorname{Vmax} /(\mathrm{KM}+\mathrm{C})) * \mathrm{C} / \mathrm{V}+\mathrm{Q} / \mathrm{V} *\left(\mathrm{C}_{2}-\mathrm{C}\right)
\end{array}
$$

where $\mathrm{C}$ is concentration in the central compartment, $\mathrm{C}_{2}$ is the concentration in the peripheral compartment, $\mathrm{CL}$ is the linear clearance term prior to time $=\alpha, \eta$ is the linear clearance term after time $=\alpha, \alpha$ is the estimated point at which the clearance term changes, $\mathrm{V}_{\max }$ is the maximum nonlinear elimination rate, $\mathrm{KM}$ is the Michaelis-Menten constant, $\mathrm{V}$ is the volume of distribution for the central compartment, and $\mathrm{Q}$ is the intercompartmental clearance.

\section{Statistical Analyses}

Following two-compartmental analysis, the estimated drug clearance change and the associated time point were used to correlate with the time of onset and magnitude of ADA. A good correlation suggests that the cause of the impact on PK may be due to ADA formation. The non-linear mixed effects hierarchical model was used, whose structure is described above. The concentrations were assumed to follow a log-normal distribution, and the individual PK parameters were also assumed to follow a multivariate log-normal 
distribution. The model was fitted using the Bayesian Markov Chain Monte Carlo (MCMC) method, and WinBugs was used to perform the analysis as described earlier.

If $\theta_{\mathrm{i}}$ represents a vector of individual $\mathrm{PK}$ parameters, then it was assumed that they follow a distribution with population parameters $\Theta$. The parameters $\Theta$ were then assigned uninformative prior distributions. The Bayesian analysis involved the estimation of the joint distribution of all parameters conditional on the observed data: $p(\theta, \Theta \mid \mathrm{PK}$ data), where $\theta$ denotes collections of all individual specific PK parameters. Generating random samples from the joint posterior distribution allows the marginal distribution of each parameter to be completely characterized. A more detailed information on Bayesian analyses of PK-PD models may be found in Lunn et al. (14) and Duffull et al. (15). The model was fit to the data using the software WinBugs v1.4.3 (http:// www.mrc-bsu.cam.ac.uk/bugs/welcome.shtml) with the Pharmaco interface and WBDiff, which together make up PKBugs v2.0 (http://winbugs-development.mrcbsu.cam.ac.uk/). The differential equations were hardwired into the compiler for WinBugs, Blackbox v1.6 (http:// www.oberon.ch/blackbox.html), in order to speed up the run time of the programs. The code on the programs can be made available on request. The convergence was assessed both visually, by examining trace and running quartile plots, and formally using the Brooks-Gelman-Rubin diagnostic available in WinBugs (16). The study was conducted with five monoclonal antibody clones that were analyzed together in a single hierarchical model, with the clones representing an additional level in the hierarchy. This means that the individual PK parameters followed a distribution based on the specific clone (with parameters associated with the particular clone). Then, the parameters from each clone were assumed to follow a common distribution with population parameters. This approach is similar to what is done in a model-based meta-analyses. The data from the clones were also analyzed separately, producing similar results. The advantage of assessing the clones together is that the model parameters are still generated for each clone, but they are estimated with greater precision. This is useful here since there are a small number of animals dosed with each clone.

\section{RESULTS}

Using the observed concentration-time data of five mAbs from a single dose PK study in which the intravenous infusion was administered into cynomolgus monkeys, the potential impact of immune response on $\mathrm{PK}$ of $\mathrm{mAb}$ was investigated.

\section{Concentration-Time Profiles of Five mAb Clones}

Serum samples were analyzed for quantification of mAbs using the method described above. Individual concentrationtime profiles of the five mAbs are presented in Fig. 1.

From the concentration-time profiles, three distinct categories of profiles were identified that were not necessarily specific to any of the five clones. First group of profiles (Category A) appeared to have a faster terminal half-life starting around day 10 $(240 \mathrm{~h})$, the second group (Category B) started to clear after day $14(336 \mathrm{~h})$, and the third group (Category C) had measurable levels of mAbs until day $35(840 \mathrm{~h})$ after the IV administration. To

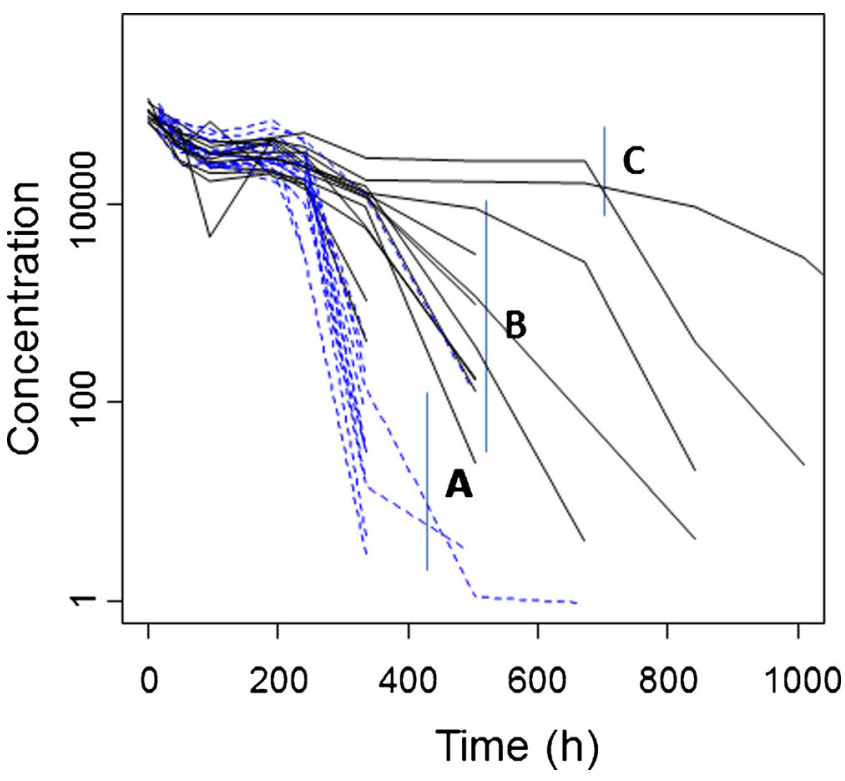

Fig. 1. Concentration $(\mathrm{ng} / \mathrm{mL})$-time $(\mathrm{h})$ profiles $(N=30)$ of the observed data including five different $\mathrm{mAb}$ clones $(N=6$ per clone $)$ after the single intravenous administration of $4 \mathrm{mg} / \mathrm{kg} \mathrm{mAbs}$. Blue dotted lines represent detection of anti-drug antibodies (ADAs) at $336 \mathrm{~h}$ post-dose. Black lines represent the detection of ADA after $336 \mathrm{~h}$ post-dose. Three distinct categories of profiles were identified as A, B, and C

determine if these categories are associated with a specific magnitude and onset of immune responses, the immunogenicity assessment was performed in serum samples collected on predose time points and day $7(168 \mathrm{~h})$, day $14(336 \mathrm{~h})$, day $21(504 \mathrm{~h})$, and day $28(672 \mathrm{~h})$ of all animals.

\section{Characterization of ADA Response}

Table I summarizes the immune responses to the five mAbs. The incidence, onset of immune response, magnitude, and specificity towards the CDR and Fc were also evaluated. Of the 30 animals evaluated, 28 animals (93\%) developed ADA responses to their administered $\mathrm{mAbs}$ (Table I). The blue dotted lines represent the animals with ADAs detected at $336 \mathrm{~h}$, and black solid lines represent animals with ADA detected after $336 \mathrm{~h}$ or where no ADA was detected (Fig. 1). All ADA positive $(n=28)$ animals belonged to categories $\mathrm{A}$ and $\mathrm{B}$ while two ADA negative animals were placed in category $\mathrm{C}$. The variable profiles associated with the categories $\mathrm{A}$ and $\mathrm{B}$ compared to category $\mathrm{C}$ revealed that immune response could be associated with change in clearance. To determine how the varying degree of immune response can affect the change in clearance, the characteristics of ADAs were further explored.

Both categories A and B showed an onset of immune response as early as day $14(336 \mathrm{~h})$. An increase in magnitude of ADA response was also observed with progression of time from day $14(336 \mathrm{~h})$ to day $21(504 \mathrm{~h})$ and finally to day 28 $(672 \mathrm{~h})$. The specificity assessment (data not shown) confirmed that the predominant reactivity was against the CDR region of their respective $\mathrm{mAbs}$ for $\mathrm{mAb} 1$ and $\mathrm{mAb} 3$ and against the CDR and $\mathrm{Fc}$ regions for $\mathrm{mAbs} 2,4$, and 5. The magnitude and incidences of the immune responses were much lower for the animals dosed with clones 2 and 4. A subset of animals in both categories $\mathrm{B}$ and $\mathrm{C}$ was associated with a later onset of immune response and were associated 
Table I. The Onset and Magnitude of an Immune Response or AntiDrug Antibodies in Cynomolgus Monkeys to Their Respective mAbs Administered on Days 14 (336 h), 21 (504 h), and 28 (672 h)

\begin{tabular}{|c|c|c|c|c|}
\hline \multirow[b]{2}{*}{ Animal number } & \multirow[b]{2}{*}{ Hum Ab clone } & $336 \mathrm{~h}$ & $504 \mathrm{~h}$ & $672 \mathrm{~h}$ \\
\hline & & \multicolumn{3}{|c|}{$\mathrm{S} / \mathrm{N}$ result* } \\
\hline V001970 & 1 & 41.07 & 207.99 & 324.21 \\
\hline V001776 & 1 & 100.01 & 234.07 & 379.61 \\
\hline V001977 & 1 & 76.92 & 307.15 & 465.08 \\
\hline V001980 & 1 & 122.62 & 110.07 & 181.54 \\
\hline V001997 & 1 & 1 & 4.39 & 91.92 \\
\hline V001993 & 1 & 44.31 & 129.16 & 208.36 \\
\hline V001672 & 2 & 1 & 6.94 & 18.22 \\
\hline V001775 & 2 & 1.71 & 16.07 & 26.12 \\
\hline V002000 & 2 & 1 & 1.95 & 8.87 \\
\hline V001830 & 2 & 7.87 & 27.07 & 26.67 \\
\hline V001853 & 2 & 1 & 1.82 & 9.22 \\
\hline V001992 & 2 & 1 & 4.24 & 12.33 \\
\hline V001968 & 3 & 60.89 & 158.69 & 402.84 \\
\hline V001975 & 3 & 13.1 & 120.2 & 265.95 \\
\hline V001984 & 3 & 40.79 & 242.99 & 419.88 \\
\hline V001774 & 3 & 82.92 & 345.64 & 513.61 \\
\hline V001756 & 3 & 93.57 & 525.55 & 691.52 \\
\hline V002003 & 3 & 23.56 & 246.8 & 445.15 \\
\hline V001769 & 4 & 1 & 1 & 1 \\
\hline V001855 & 4 & 1 & 1 & 1 \\
\hline V002002 & 4 & 23.84 & 267.87 & 407.32 \\
\hline V001785 & 4 & 1 & 2.02 & 11.02 \\
\hline V001849 & 4 & 1 & 1 & 1.67 \\
\hline V001982 & 4 & 1 & 7.52 & 310.22 \\
\hline V001971 & 5 & 1 & 22.45 & 159.12 \\
\hline V001978 & 5 & 1 & 3.77 & 105.54 \\
\hline V001979 & 5 & 1 & 6.93 & 176.78 \\
\hline V001841 & 5 & 11.32 & 114.65 & 333.45 \\
\hline V001983 & 5 & 2.32 & 31.33 & 176.97 \\
\hline V001994 & 5 & 31.36 & 115.3 & 354.39 \\
\hline
\end{tabular}

Note: Samples from pre-dose and day 7 were analyzed for detection of ADA and these time points were ADA negative for all animals *Signal to noise $(\mathrm{S} / \mathrm{N})$ is the ratio of the raw electrochemiluminescence read of the post dose sample and the pre dose sample

with a lower S/N (Table I, animals dosed with clones 2 and 4) and were also associated with TP levels in the 100-1000 ng/ $\mathrm{mL}$ range till $800 \mathrm{~h}$.

\section{Evaluation of PK Parameter Estimates Using Non-Compartmental and Single-Compartmental Analyses}

The PK parameter estimates were evaluated for ADA positive $(N=28)$ and negative $(N=2)$ animals to understand the impact of immunogenicity on mAb levels. The PK parameters and estimates were obtained using both noncompartmental analysis (NCA) and one-compartmental model for TP concentration-time data of individual animals. Although there were not enough ADA negative animals, it was obvious that ADA appears to impact the estimation of PK parameters. The area under the curves (AUCs) in ADA positive animals decreased by $>50 \%$ compared to that of ADA negative animals regardless of the PK model (NCA Vs one compartment) used. The mean $t_{1 / 2}( \pm \mathrm{SD})$ was $50.6 \pm 24.9 \mathrm{~h}$ for ADA negative animals and 42.4 $\pm 35.6 \mathrm{~h}$ for ADA positive animals (Table II) using the NCA model. The time at the last measureable concentration $\left(\mathrm{T}_{\text {last }}\right) \%$ in ADA positive animals $(N=28)$ was also reduced to $424 \mathrm{~h}(17.7$ days $)$ compared to $1090 \mathrm{~h}$ (45.5 days) in ADA negative animals $(N=2)$.

The time at the last measureable concentration $\left(\mathrm{T}_{\text {last }}\right)$ was also reduced by $61 \%$ in ADA positive animals. The clearance increased by 2.2-fold in ADA positive animals in both models indicating an ADA-mediated enhanced clearance. Interestingly, with the one-compartment model, halflife of mAbs in central compartment for ADA positive animals was 2.6-fold shorter than ADA negative animals. In addition, the concentration-time profile also suggested that the decrease in AUC, half-life, and $\mathrm{T}_{\text {last }}$ was not due to target or non-specific FcRn mediated mechanisms. Clearly, the presence of ADAs appeared to be associated with a steep drop in mAb concentrations, due to an increase in the clearance. However, such a decline in $\mathrm{mAb}$ concentrations associated with a simultaneous onset of ADA and a consequent increase in clearance might differ for each individual animal. This was evident from the high variability among ADA positive animals for all parameter estimates. Hence, an additional parameter that evaluated the change in clearance time was explored through a statistical modeling approach.

Table II. A Non-Compartmental and Single Compartmental Analyses of PK Parameter Estimates in ADA Positive and Negative Groups from Single Dose Study

\begin{tabular}{|c|c|c|c|c|c|c|c|c|c|}
\hline & & \multicolumn{5}{|l|}{$\mathrm{NCA}$} & \multicolumn{3}{|l|}{ One compartment } \\
\hline \multicolumn{2}{|c|}{ ADA grouping } & $\mathrm{T}_{1 / 2}(\mathrm{~h})$ & $\mathrm{T}_{\text {last }}(\mathrm{h})$ & $\mathrm{C}_{\text {last }}(\mu \mathrm{g} / \mathrm{mL})$ & $\operatorname{AUC}_{0-\text { inf }}(\mathrm{h} * \mu \mathrm{g} / \mathrm{mL})$ & $\mathrm{Cl}(\mathrm{mL} / \mathrm{h} / \mathrm{kg})$ & AUC $(\mathrm{h} * \mu \mathrm{g} / \mathrm{mL})$ & K10_HL (h) & $\mathrm{CL}(\mathrm{mL} / \mathrm{h} / \mathrm{kg})$ \\
\hline \multirow[t]{4}{*}{ Negative } & $\mathrm{N}$ & 2 & 2 & 2 & 2 & 2 & 2 & 2 & 2 \\
\hline & Mean & 50.6 & 1090 & 0.170 & 25,200 & 0.160 & 29,400 & 287 & 0.139 \\
\hline & SD & 24.9 & 119 & 0.208 & 3510 & 0.022 & 5730 & 39.2 & 0.027 \\
\hline & CV\% & 49.2 & 10.9 & 122 & 13.9 & 13.9 & 19.5 & 13.6 & 19.5 \\
\hline \multirow[t]{4}{*}{ Positive } & $\mathrm{N}$ & 28 & 28 & 28 & 28 & 28 & 28 & 28 & 28 \\
\hline & Mean & 42.4 & 424 & 2.42 & 11,700 & 0.358 & 13,300 & 111 & 0.323 \\
\hline & SD & 35.6 & 167 & 5.47 & 2730 & 0.080 & 3980 & 33.5 & 0.080 \\
\hline & $\mathrm{CV} \%$ & 84.1 & 39.3 & 226 & 23.2 & 22.3 & 30.0 & 30.2 & 24.4 \\
\hline
\end{tabular}

$N C A$ non-compartmental analysis, $T_{1 / 2}$ half-life, $T_{\text {last }}$ time at last measurable concentration, $C_{\text {last }}$ concentration at last measurable time point, $A U C$ area under a concentration-time curve, $A U C 0$-inf AUC from time 0 to infinity, $C L$ clearance, $K 10$ the rate at which the drug leaves the system from the central compartment. The elimination rate K10_HL: the half-life associated with the rate constant K10 


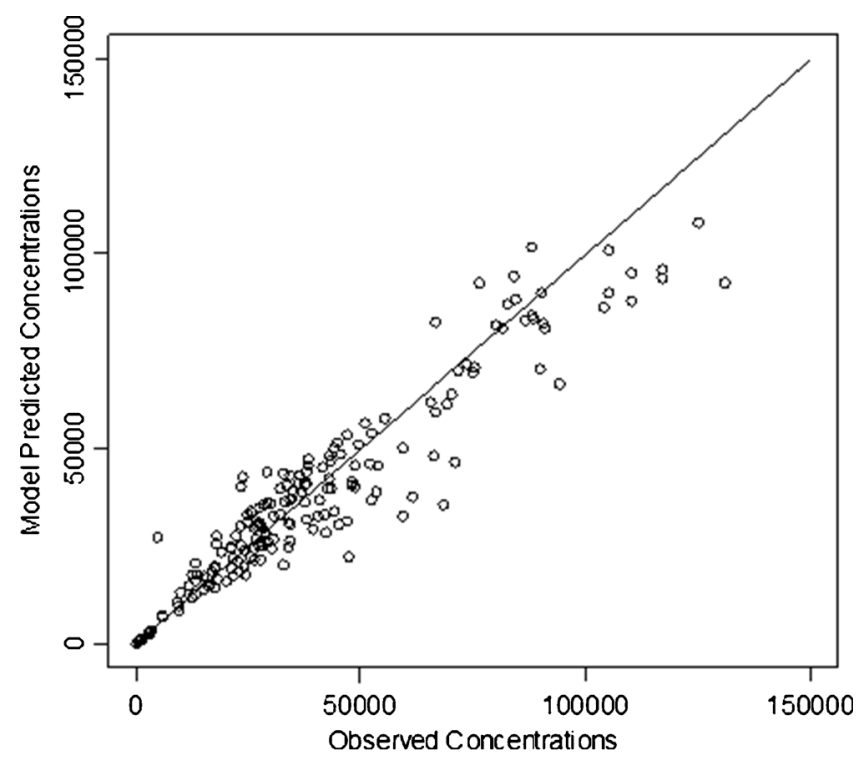

Fig. 2. Observed mAbs concentrations ( $x$-axis) versus modelpredicted concentrations ( $y$-axis) based on medians from Bayesian posterior simulations

\section{Modeling to Predict Time of Clearance Change}

Simplified two-compartment target-mediated disposition model derived from a version of previously described population PK model was used to predict time of clearance change, a parameter that cannot be determined from NCA and one-compartmental models. This two-compartmental statistical model utilized the change in clearance and the time at which the clearance changed as parameters to predict clearance of the mAbs and was built on the hypothesis that change in clearance and time at which the clearance changed could be predicted if the exposure is impacted.

Figure 2 shows the actual concentrations versus the model-predicted concentrations to further illustrate that the model fits well. Figure $3 \mathrm{a}-\mathrm{f}$ shows the individual fits for six animals, five from ADA positive animals (a-e), where one animal from each clone was used in the model. The last animal (f) was one of two animals who did not develop ADA. These results indicated that the model fits well in individual animals. Figure 4a shows the actual time of ADA detection versus estimated time of clearance change for ADA positive and negative animals. Interestingly, the estimated time at a

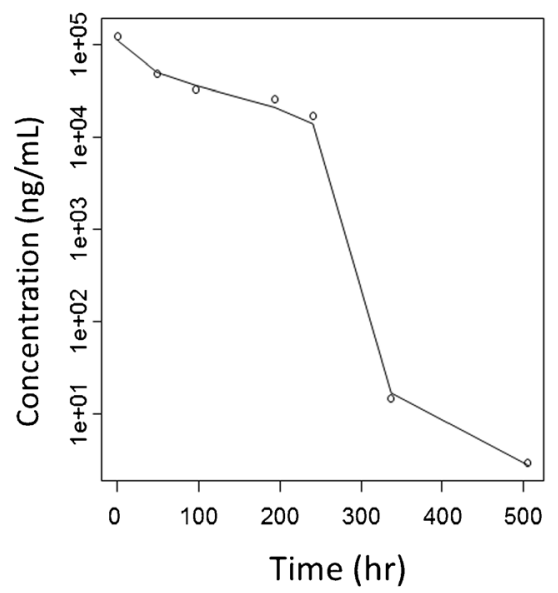

d

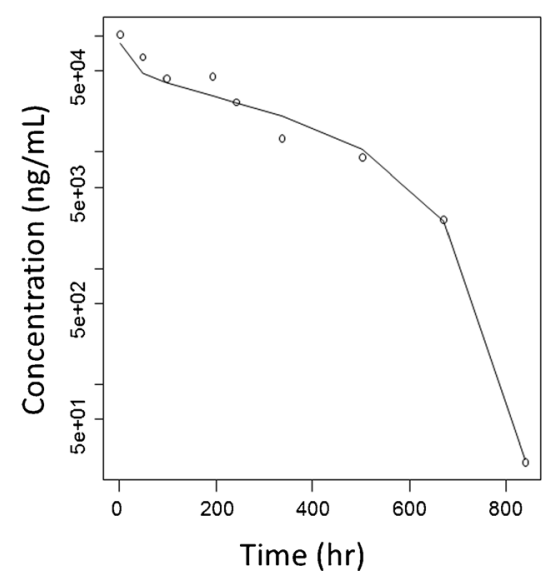

b

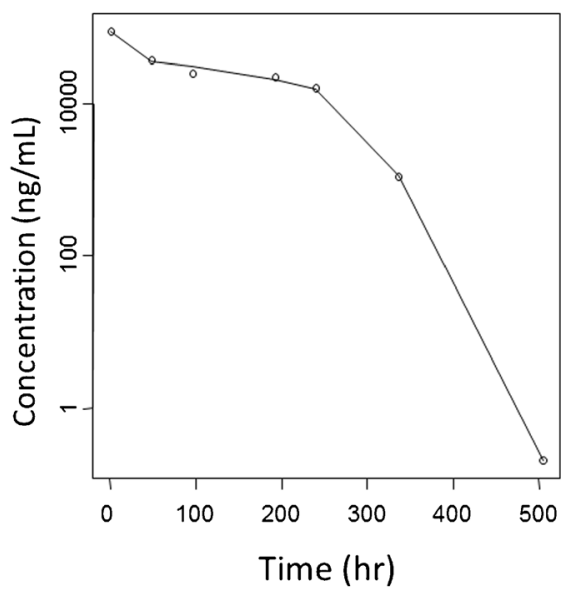

e

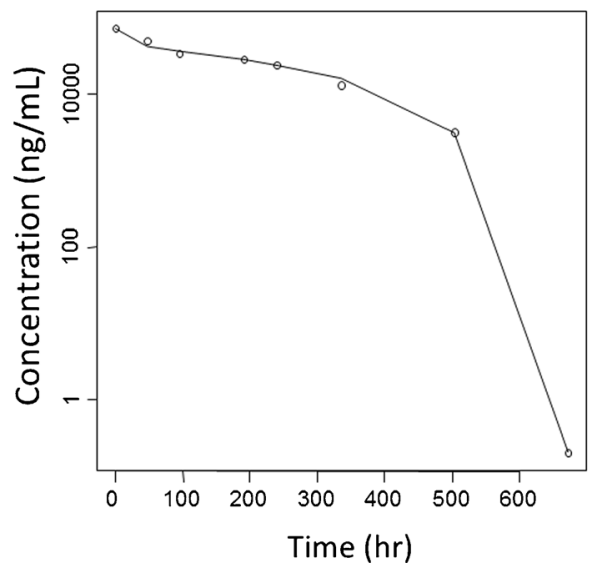

C

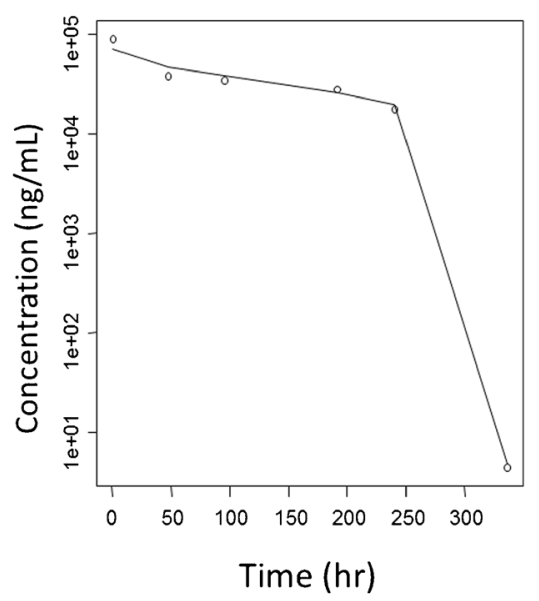

f

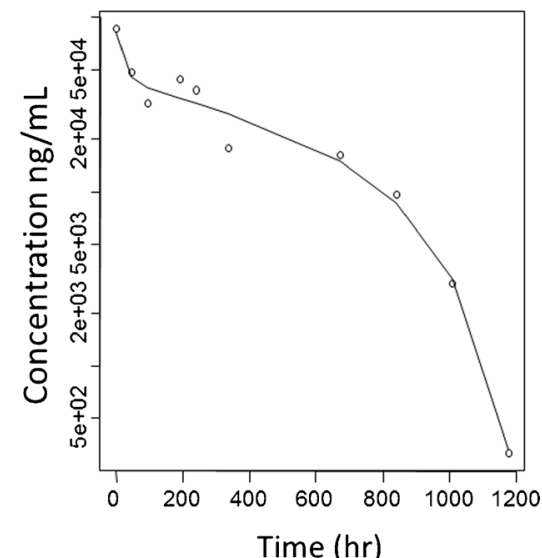

Fig. 3. Example model fit plots (concentration time) for six individual animals. The first five figures (a-e) were animals, one from each clone, who developed ADA. The last figure (f) is an animal that did not develop ADA 
a

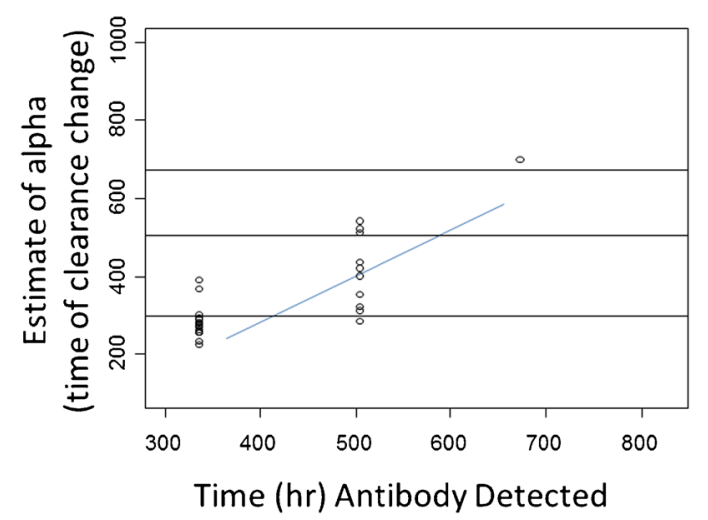

b
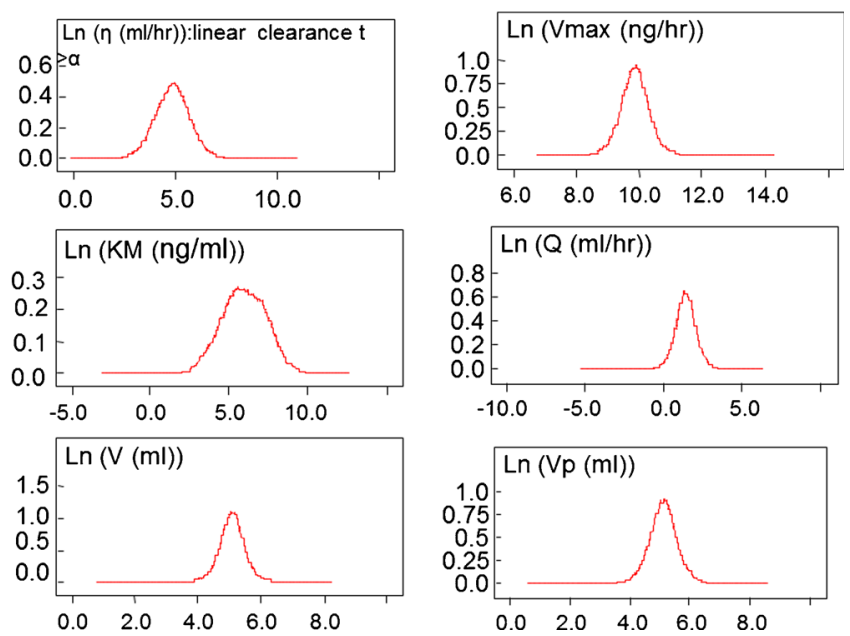

Fig. 4. a Time the antibodies were detected versus the estimated time of linear clearance change (median of the posterior distribution). b Posterior distributions for the natural $\log$ of population mean parameters; $\eta$ is the linear clearance term after time $=\alpha, V_{\max }$ maximum non-linear elimination rate, $K M$ Michaelis-Menten constant, $Q$ intercompartmental clearance, $V$ volume of distribution for the central compartment, and $V_{p}$ volume of distribution for the peripheral compartment)

which the clearance changed ( $\alpha$ on $y$-axis) was close to or before the actual time (h) at which ADA were detected ( $x$ axis) in most instances indicating that estimated time at which the clearance changed could be associated with the onset of ADA in an individual animal. There were two animals that did not have detectable ADAs until day $28(672 \mathrm{~h})$ in this study. This two-compartment model predicted that one ADA negative animal had an estimated time for clearance change at $767 \mathrm{~h}$ while the last sample collected and analyzed for ADA was $672 \mathrm{~h}$; approximately 4 days prior to the estimated time of clearance change suggesting that onset of ADA may be later than the sample analyzed for ADA detection. This indicates that model can predict the presence of ADA even though the sample was bioanalytically negative due to potential interference by excess TP. Additionally, this model also predicted that other ADA negative animal had a probability of 0.95 that $\mathrm{mAB}$ clearance did not change during the course of the study, indicating this animal was ADA negative. Additionally, the Bayesian framework allows for the estimation of probability distributions for the parameters of the model. The posterior distribution for some of the population parameters $(\eta$ : linear clearance at second compartment, $\mathrm{V}_{\max }$ : maximum non-linear elimination rate, $\mathrm{KM}$ : Michaelis-Menten constant, Q: intercompartmental clearance, $\mathrm{V}$ : volume of distribution for the central compartment, and Vp: volume of distribution for the peripheral compartment) from the two-compartmental simplified target-mediated disposition model of all animals including ADA negative animals is shown in Fig. 4b and summarized in Table III. The CL, linear portion of the clearance prior to time $\alpha$, the estimated point at which the clearance term changes, was removed from the model since the compound is not readily cleared until the development of ADA or the target-mediated clearance takes effect after the distribution phase. In this case, the linear portion of the clearance could not be reliably estimated. They were given priors to prevent them from being zero, but in this case, the compound was not readily cleared until the development of ADA or target-mediated clearance took effect. This is not always the case though as this parameter has been estimated for analyses with other dataset. A posterior probability distribution of $\alpha$ was estimated for all animals including ADA negative animals. An example based on one of the ADA positive animals is shown in Fig. 5 (Animal No. 4). The $y$-axis represents the posterior probability distribution of $\alpha$ and $x$-axis represents the time (h). This particular animal had a probability of 0.92 that the linear term of the clearance changed $(\alpha)$ while on the study. The onset of ADA detected by UNISA in this animal was at $336 \mathrm{~h}$ (Table I, Animal No. 4), while the model estimated time to have an ADA-mediated clearance change is about $240 \mathrm{~h}$ (Fig. 5). The $\mathrm{mAb}$ levels in the serum at $240 \mathrm{~h}$ were about $17 \mu \mathrm{g}$ that could have limited the detection of ADA at that timepoint. However, the results here suggested that with a high probability, the animal's clearance was affected well before the actual detection of ADA. Similar figures were generated for all animals but are not shown in this article. The summary statistics of the posterior distribution of $\alpha$ were as follows: median: $282 \mathrm{~h}(240 \mathrm{~h}$ and $449 \mathrm{~h}$ are 2.5 and 97.5 percentiles respectively). These data suggested that the time at which the linear term of the clearance changes would occur between 10 and 18 days following a single dose.

\section{DISCUSSION}

PK modeling and simulation at early phases of clinical development is utilized in order to understand dose-exposure response relationships of therapeutic proteins (17-20). The primary use of the PK modeling in preclinical studies is to determine: (i) the PK parameters for the therapeutic proteins, (ii) maximal tolerated doses through exposure level analysis in preclinical species, and (iii) the exposure margin for clinical subjects as well as to predict human dose for therapeutics. Currently, the evaluation of exposure estimates in preclinical species involves elimination of individual ADA positive animals from such analysis if the presence of ADA decreases AUC by an arbitrary number of greater than 2 -fold. The goal of this study was to develop statistically predefined criteria for selection of subjects for PK analysis.

The dose selection for first-in-human and prediction of human PK are based on preclinical PK data. PK parameters 
Table III. Summary of Posterior Distribution Parameters from the Two-Compartmental Simplified Target-Mediated Disposition Model

\begin{tabular}{|c|c|c|c|c|c|c|c|}
\hline \multirow[b]{2}{*}{ Clone(s) } & \multicolumn{5}{|c|}{ Two-compartment parameter estimates (units) } & \multirow[b]{2}{*}{$\mathrm{Km}(\mathrm{ng} / \mathrm{mL})$} & \multirow[b]{2}{*}{$\operatorname{Vmax}(\mathrm{ng} / \mathrm{h})$} \\
\hline & $\mathrm{Vc}=\mathrm{V} 1(\mathrm{~mL})$ & $\mathrm{V} 2=\mathrm{Vp}(\mathrm{mL})$ & Eta $(\mathrm{mL} / \mathrm{h})$ & $\mathrm{Q}(\mathrm{mL} / \mathrm{h})$ & & & \\
\hline \multirow[t]{4}{*}{ All } & $\mathrm{N}$ & 30 & 30 & 30 & 30 & 30 & 30 \\
\hline & Geometric mean & 162 & 167 & 130 & 4.01 & 380 & 19,000 \\
\hline & Median & 162 & 167 & 130 & 4.01 & 3780 & 19,000 \\
\hline & $5 \%, 95 \%$ & $82.3,317$ & $73.0,372$ & $31.5,540$ & $1.31,12.8$ & $35.9,3600$ & $8600,41,000$ \\
\hline \multirow[t]{4}{*}{1} & $\mathrm{~N}$ & 5 & 5 & 5 & 5 & 5 & 5 \\
\hline & Geometric mean & 125 & 122 & 156 & 6.17 & 317 & 21,100 \\
\hline & Median & 125 & 123 & 151 & 6.11 & 314 & 21,600 \\
\hline & $5 \%, 95 \%$ & $75.9,206$ & $60.3,233$ & $27.1,1050$ & $1.91,20.5$ & $18.5,5120$ & $10,400,39,300$ \\
\hline \multirow[t]{4}{*}{2} & $\mathrm{~N}$ & 5 & 5 & 5 & 5 & 5 & 5 \\
\hline & Geometric mean & 169 & 219 & 90.0 & 9.58 & 455 & 24,600 \\
\hline & Median & 172 & 221 & 92.8 & 8.50 & 513 & 25,300 \\
\hline & $5 \%, 95 \%$ & $97.5,284$ & 111,416 & $19.5,376$ & $3.19,42.5$ & $27.9,5110$ & $11,800,46,200$ \\
\hline \multirow{4}{*}{3} & $\mathrm{~N}$ & 5 & 5 & 5 & 5 & 5 & 5 \\
\hline & Geometric mean & 180 & 204 & 255 & 1.79 & 213 & 16,500 \\
\hline & Median & 180 & 200 & 268 & 1.77 & 209 & 17,500 \\
\hline & $5 \%, 95 \%$ & 108,300 & $79.8,578$ & $47.9,1130$ & $0.52,6.62$ & $14.0,3530$ & $6310,35,200$ \\
\hline \multirow[t]{4}{*}{4} & $\mathrm{~N}$ & 5 & 5 & 5 & 5 & 5 & 5 \\
\hline & Geometric mean & 178 & 183 & 94.6 & 3.67 & 503 & 14,900 \\
\hline & Median & 178 & 187 & 96.5 & 3.67 & 561 & 15,200 \\
\hline & $5 \%, 95 \%$ & 110,284 & 100,321 & $24.1,354$ & $1.48,9.21$ & $57.4,3170$ & $7700,27,700$ \\
\hline \multirow[t]{4}{*}{5} & $\mathrm{~N}$ & 5 & 5 & 5 & 5 & 5 & 5 \\
\hline & Geometric mean & 161 & 123 & 108 & 2.91 & 539 & 19,100 \\
\hline & Median & 161 & 125 & 116 & 2.89 & 503 & 19,500 \\
\hline & $5 \%, 95 \%$ & 100,257 & $59.7,240$ & $22.0,424$ & $1.04,8.58$ & $64.1,5010$ & $9700,34,900$ \\
\hline
\end{tabular}

of Cmax and AUC (exposure) in these preclinical studies could be impacted by ADA. The impact of such ADA on PK parameters exposure analysis can vary depending on the characteristics of ADA in individual subjects.

In this study, we evaluated the PK parameters and induction of ADA of five variants (clones). Each clone was associated with a different onset of response time (h) and magnitude (S/N). While clones 1 and 3 elicited a high level of response in majority of dosed animals within $336 \mathrm{~h}$ (Table I), some animals from clones 2 and 4 were associated with magnitudes lower than 50 and a TP level between 800 and $1000 \mathrm{ng} / \mathrm{mL}$ (Fig. 1). The variability of the clones was most likely due to the differences in CDR and was an important criteria for the design of the modeling study. The differences

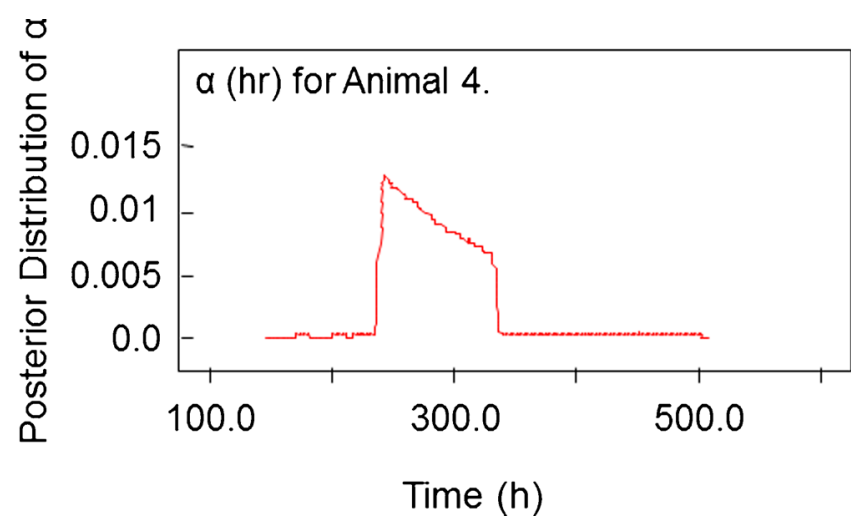

Fig. 5. Posterior distribution of $\alpha$ (time at which the linear term of the clearance changes) for Animal 4 in CDRs also indicated different affinities to the target. These differences in the clones were critical to develop a model that could differentiate impact of ADA versus target-mediated disposition of the TP.

The first component of the model was to include data on characterization of the ADA. The severity of impact of ADA on PK parameters is dependent on the nature of the ADA (i.e., the magnitude measured as titer or concentration, onset time, transient versus persistent). In this study, the mathematical model faithfully predicted the change in clearance time $(\alpha)$ based on observed concentration-time profile of five different $\mathrm{mAb}$ clones that can correlate with formation of ADAs.

A second component of the modeling exercise required the accurate measurement of PK and ADA levels during bioanalytical analysis $(5,21)$. The influence of high levels of TP limit detection of ADA, and high levels of ADA interfere with accurate measurement of TP. In this study, we utilized specific and sensitive assays for the bioanalytical measurements of PK and ADA levels that have minimal interference from matrix associated factors using the approach of integrating the "free" and "total" TP detections (Thway et al. $(4,22,23))$. Further accuracy of the ADA and TP measurements requires a comprehensive evaluation of interference due to circulating ligands (soluble form or shed form of membrane receptors), matrix associated factors like excess TP or ADA, biodegraded or transformed fragments as in fusion proteins and small peptides or toxins as in antibody drug conjugates $(24,25)$. These analyses ensured the accuracy of the PK and ADA data in generation of the model. A third component built into the model to assess the impact of ADA 
on PK was the impact of various factors that contribute to the impact on exposure. Immune response to a TP is interplay of several factors that include cells involved in antigen processing, sequence of proteins, genetic and diseased state of individuals, and concomitant medications (7-9). In this study, animal weights, time of administration, and other demographic parameters were included along with real time PK data from preclinical studies. By estimating the $\alpha$ or change in time of clearance using data from a single dose PK study, it could be predicted that this parameter was different for ADA positive animals when compared to ADA negative animals. The change in clearance ( $\eta$ ) may also be useful to evaluate the impact that the ADA has on the clearance. However, sampling frequency should be considered during interpretation of these data. In summary, we have developed a novel modeling approach to predict the impact of ADA on PK using a non-compartmental as well as one-compartmental analysis. The model was able to (i) faithfully predict time of clearance change, a parameter not determined from NCA and one-compartmental models, and (ii) apply the posterior distribution for some of the population parameters to determine the likelihood of ADA impact on PK in the absence of ADA data. The utility of this modeling approach also provides an alternate method of analysis of impact, due to the challenges of accuracy of ADA and TP measurements due to high circulating drug and/or ADA during the dosing phase. With the new ICH S6 guidance, where the ADA assessment is limited to animals with abnormal PK or toxicology findings, this proposed model can use the time of clearance change parameters to delineate the impact due to ADA on PK profiles of dosed animals.

\section{REFERENCES}

1. Shi S. Biologics: an update and challenge of their pharmacokinetics. Curr Drug Metab. 2014;15(3):271-90.

2. Luu KT et al. A model-based approach to predicting the human pharmacokinetics of a monoclonal antibody exhibiting targetmediated drug disposition. J Pharmacol Exp Ther. 2012;341(3):702-8.

3. Chirmule N, Jawa V, Meibohm B. Immunogenicity to therapeutic proteins: impact on PK/PD and efficacy. AAPS J. 2012;14(2):296-302.

4. Thway TM et al. Impact of anti-drug antibodies in preclinical pharmacokinetic assessment. AAPS J. 2013;15(3):856-63.

5. Sailstad JM et al. A white paper-consensus and recommendations of a global harmonization team on assessing the impact of immunogenicity on pharmacokinetic measurements. AAPS J. 2014;16(3):488-98.

6. Perez Ruixo JJ, Ma P, Chow AT. The utility of modeling and simulation approaches to evaluate immunogenicity effect on the therapeutic protein pharmacokinetics. AAPS J. 2013;15(1):17282.

7. Chen $\mathrm{X}$ et al. A mathematical model of the effect of immunogenicity on therapeutic protein pharmacokinetics. AAPS J. 2013;15(4):1141-54.

8. Chen X, Hickling TP, Vicini P. A mechanistic, multiscale mathematical model of immunogenicity for therapeutic proteins: part 2-model applications. CPT Pharmacometrics Syst Pharmacol. 2014:3:e134.

9. Chen X, Hickling TP, Vicini P. A mechanistic, multiscale mathematical model of immunogenicity for therapeutic proteins: part 1-theoretical model. CPT Pharmacometrics Syst Pharmacol. 2014;3:e133.

10. Bonate PL. Recommended reading in population pharmacokinetic pharmacodynamics. AAPS J. 2005;7(2):E363-73.

11. Bonate PL. Covariate detection in population pharmacokinetics using partially linear mixed effects models. Pharm Res. 2005;22(4):541-9.

12. Bonate PL et al. The distribution, metabolism, and elimination of clofarabine in rats. Drug Metab Dispos. 2005;33(6):739-48.

13. Мa P. Theoretical considerations of target-mediated drug disposition models: simplifications and approximations. Pharm Res. 2012;29(3):866-82.

14. Lunn DJ et al. Bayesian analysis of population PK/PD models: general concepts and software. J Pharmacokinet Pharmacodyn. 2002;29(3):271-307.

15. Duffull SB et al. Analysis of population pharmacokinetic data using NONMEM and WinBUGS. J Biopharm Stat. 2005;15(1):53-73.

16. Gelman A, Carlin JB, Stern HS, Rubin DB. Bayesian data analysis, in Bayesian data analysis. 2003, Chapman and Hall/CRC.

17. Singh SS. Preclinical pharmacokinetics: an approach towards safer and efficacious drugs. Curr Drug Metab. 2006;7(2):165-82.

18. U.S. Department of Health and Human Services Food and Drug Administration, C.f.D.E.a.R.C., Center for Veterinary Medicine (CVM). Guidance for Industry Bioanalytical Method Validation. [PDF] 2001; Available from: www.fda.gov/downloads/drugs/ GuidanceComplianceRegulatoryInformation/Guidances/ ucm070107.pdf.

19. Davda JP et al. A model-based meta-analysis of monoclonal antibody pharmacokinetics to guide optimal first-in-human study design. MAbs. 2014;6(4):1094-102.

20. Dong JQ et al. Quantitative prediction of human pharmacokinetics for monoclonal antibodies: retrospective analysis of monkey as a single species for first-in-human prediction. Clin Pharmacokinet. 2011;50(2):131-42.

21. Kelley M, DeSilva B. Key elements of bioanalytical method validation for macromolecules. AAPS J. 2007;9(2):E156-63.

22. Kelley $\mathrm{M}$ et al. Theoretical considerations and practical approaches to address the effect of anti-drug antibody (ADA) on quantification of biotherapeutics in circulation. AAPS J. 2013;15(3):646-58.

23. Lee JW et al. Bioanalytical approaches to quantify "total" and "free" therapeutic antibodies and their targets: technical challenges and PK/PD applications over the course of drug development. AAPS J. 2011;13(1):99-110.

24. Kaur S et al. Bioanalytical assay strategies for the development of antibody-drug conjugate biotherapeutics. Bioanalysis. 2013;5(2):20126.

25. Chen B et al. Pharmacokinetics/pharmacodynamics modelsupported early drug development. Curr Pharm Biotechnol. 2012;13(7):1360-75. 